\title{
Self-reconfiguration of Modular Underwater Robots using an Energy Heuristic
}

\author{
Furno, Lidia; Blanke, Mogens; Galeazzi, Roberto; Christensen, David Johan
}

Published in:

Proceedings of 2017 IEEE/RSJ International Conference on Intelligent Robots and Systems

Link to article, DOI:

10.1109/IROS.2017.8206530

Publication date:

2017

Document Version

Peer reviewed version

Link back to DTU Orbit

Citation (APA):

Furno, L., Blanke, M., Galeazzi, R., \& Christensen, D. J. (2017). Self-reconfiguration of Modular Underwater Robots using an Energy Heuristic. In Proceedings of 2017 IEEE/RSJ International Conference on Intelligent Robots and Systems (pp. 6177-6284). IEEE. https://doi.org/10.1109/IROS.2017.8206530

\section{General rights}

Copyright and moral rights for the publications made accessible in the public portal are retained by the authors and/or other copyright owners and it is a condition of accessing publications that users recognise and abide by the legal requirements associated with these rights.

- Users may download and print one copy of any publication from the public portal for the purpose of private study or research.

- You may not further distribute the material or use it for any profit-making activity or commercial gain

- You may freely distribute the URL identifying the publication in the public portal 


\title{
Self-reconfiguration of Modular Underwater Robots using an Energy Heuristic
}

\author{
Lidia Furno $^{1}$, Mogens Blanke ${ }^{1,2}$, Roberto Galeazzi ${ }^{1}$ and David Johan Christensen ${ }^{1}$
}

\begin{abstract}
This paper investigates self-reconfiguration of a modular robotic system, which consists of a cluster of modular vehicles that can attach to each other by a connection mechanism. Thereby, they can form a desired morphology to meet task specific requirements. Reconfiguration can be needed due to limitations from dimensions of passable corridors for an underwater maintenance task, for supplemental instrumentation that is available on a particular robot, or as remedial action if one robot in a cluster suffers from malfunction. Being crucial for autonomous underwater vehicles, energy consumed is employed as a heuristic. The paper shows how the Basic Theta* algorithm can be guided by an energy criterion to calculate a transition from start- to goal morphology. Individual robots are guided while minimizing the overall energy for propulsion and for balancing restoring forces and moments in morphologies. The properties of the proposed self-reconfiguration algorithm are evaluated through simulations and preliminary model tank experiments. The energy based heuristic for reconfiguration is compared to a traditional solution that minimizes the Euclidean distance.
\end{abstract}

\section{INTRODUCTION}

The use of robot technology in offshore sub-sea operations is ubiquitous and autonomous underwater vehicles (AUVs) are being routinely employed for inspection and mapping. With sub-sea technology becoming mature where the entire production plant resides at the seabed, ideas emerge that long term inspection and certain repair tasks could be made by clusters of heterogeneous robots that have individual capabilities for sensing and manipulation. Along this line, modularity properties could be added such that robots could connect to form morphologies with specific capabilities. A key problem for a swarm of such heterogeneous modular robots is the ability to autonomously reconfigure from one task specific morphology to another.

New technological solutions, which could enable underwater services with higher quality at a lower cost, are challenging fields of research and development. In this context, AUVs are rapidly gathering interest for their flexibility and anticipated potential to overcome many limitations of remotely operated vehicles (ROVs). Steps toward enhanced autonomy and use of multiple vehicles include AMOUR (Autonomous Modular Optical Underwater Robot), an underwater modular AUV for inspection and monitoring [1], and CoCoRo (Collective Cognitive Robots), a heterogeneous swarm of

1 The authors are with the Department of Electrical Engineering, Automation and Control Group, Technical University of Denmark, Elektrovej, $2800 \mathrm{Kgs}$. Lyngby, Denmark. \{furno, mb, rg, djchr\}elektro.dtu.dk

${ }^{2}$ Mogens Blanke is also affiliated with AMOS CoE, Institute of Technical Cybernetics, Norwegian University of Science and Technology, 7491 Trondheim, Norway.
AUVs used for inspection and environmental monitoring [2], [3]. AMOUR consists of a robot and an underwater sensor network that can adopt to task requirements. The robot is able to pick, transport and drop individual sensors. CoCoRo consists of three sub-systems: a floating base station that allows for localization, communication and human-robot interaction; a self-aware ground swarm of self-organizing AUVs that perform significant tasks; a relay swarm that enables communication between sub-systems. The CoCoRo system agents cannot connect with each other by means of a physical link.

Wider possibilities become available if robots are able to connect physically and form a cluster with a morphology that fulfills the requirements of any new assignment. To achieve full autonomy, such modular system need capability to self-reconfigure. The morphology is able to change to fulfill a new mission plan or to accommodate unanticipated conditions. These would include component faults or conditions in the environment. As example, the modular system could carry a tool requiring a narrow morphology to pass into a confined space; at a later time, a new task could be underwater inspection requiring robots equipped with specific sensors to be at the morphology borders. 3D visualizations of how the system configuration changes according to task requirements are shown in Figure 1. Since the energy available to each AUV is a major constraint towards longterm operation, it appears natural that a self-reconfiguration strategy must account for the energy consumption during a morphology change of the modular system.

The ability to assemble in a morphology is a distinctive feature of reconfigurable robots that results in highly versatile systems able to modify their morphology by rearranging the already existing connections [4], [5], [6]. The ability to self-reconfigure to a different morphology is illustrated by the following real-life example. Crack formation in parts of the sea bottom support structure for an offshore rig is very serious. The remedial action is to cease crack propagation by locating the crack, transport a drill to the correct position at the end of the crack line and drill a hole in the steel structure. When cracks are located in the internal part of the structure, divers cannot be employed because of access space, and working class ROVs are big and difficult to operate within a confined structure. Small and long-term reconfigurable robots would be a good alternative to monolithic robots. The modular AUVs may transport a tool to the structure, change morphology to fit the confined space while passing through a narrow passage and change morphology again to position the drill on the relevant wall. 


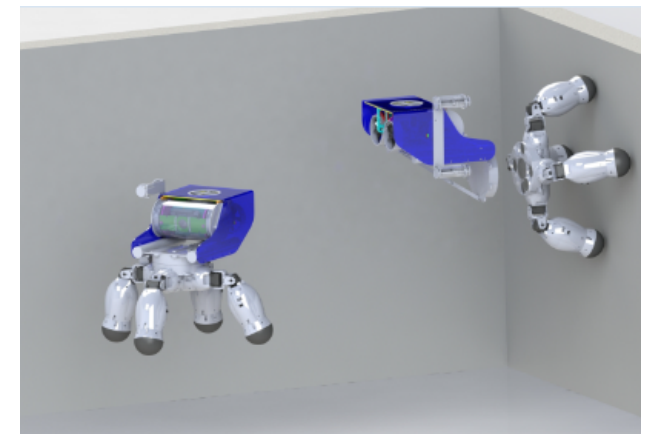

(a) Inspection of underwater structures by means of heterogeneous robots: a legged robot with relevant sensors is transported by an AUV to a suspected crack area.

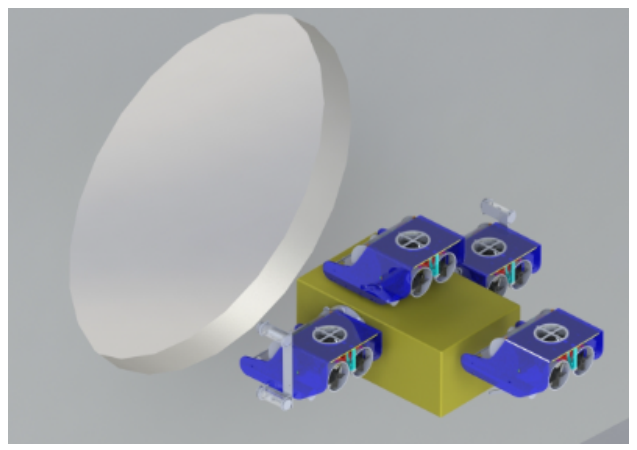

(b) Object transportation by multiple homogeneous robots through a narrow passage.

Fig. 1: Concept images depicting possible tasks.

This paper proposes a self-reconfiguration strategy for modular robotic systems that exploiting an energy heuristic determines the transitions of each agent from the start- to the goal configurations with the minimum energy consumption in all intermediate morphologies. The Basic Theta* algorithm proposed by Nash et al. [7], [8] is modified to include a cost function that accounts for both the energy spent to move the robots and the energy consumed by the cluster of robots to withstand restoring forces and moments due to morphology changes. The paper evaluates the proposed energy-based self-reconfiguration strategy in simulation and in preliminary model tank experiments. The modular underwater robotic system under development within the innovation project REMORA (REconfigurable MOdular Robotic system for Aquatic environment) is employed as a study case. Furthermore, the paper compares the proposed method with a self-reconfiguration strategy that instead has minimum Euclidean distance as a heuristic.

The paper is organized as follows: other related work is described in Section II; the REMORA platform is briefly presented in Section III; Sections IV-V provide an overview of the AUV's hydrostatics and hydrodynamics. Section VI introduces and discusses the energy criterion; whereas the self-reconfiguration problem in defined and investigate in Section VII. Lastly, results obtained through simulations and initial experiments are discussed in Section VIII.

\section{OTHER RELATED WORK}

Motion planning based on energy efficiency criteria has been widely studied in the literature [9], [10], [11], [12]. Liu et al. [11] considered an energy-related criterion as cost function and used the $\mathrm{A}^{*}$ algorithm for motion planning. Pal et al. [12] used the $A^{*}$ algorithm to generate efficient paths by adding a function of the energy consumption to an Euclidean metric, which is the cost function commonly used for motion planning. Nash et al. [7], [8] stated that the $\mathrm{A}^{*}$ algorithm generates the shortest path in the discrete environment (grids) but it is not always true in the continuous environment. Brandt [13] investigated the selfreconfiguration properties of the ATRON modular robot by applying $A^{*}$. In this case, the optimality of $A^{*}$ is recognized since ATRON is a lattice-based modular robot, which allows for movements on grids. Nash et al. proposed a variant of $\mathrm{A}^{*}$, the Basic Theta* algorithm, which finds paths closer to the optimal paths than $A^{*}$. De Filippis et al. [14] applied Basic Theta* for path generation without considering energy. Among the modular robotic systems mentioned in Section I, Detweiler et al. [15] investigated ways to save the energy of AMOUR with buoyancy and balance control.

Autonomous operations in the marine environment impose specific challenges, and various robotics platforms have been considered. The Tactically Expandable Maritime Platform (TEMP) consists of identical self-propelled robotic boats that can form connected structures autonomously [16], [17], [18]. The ANGuilliform robot with ELectric Sense robotic platform (ANGELS) is formed by nine independent rigid bodies able to connect serially to achieve bio-inspired structures [19]. Doyle et al. [20] introduced the concept of Modular Hydraulic Propulsion (MHP) in which a modular robot, intended for a fluid environment, moves by routing the fluid through itself. These modular systems are characterized by homogeneous robots docking together to provide a service. Fitch discussed different levels of heterogeneity and the limitations of homogeneous systems in [21].

\section{MODULAR PLATFORM REMORA}

REMORA [22] is an innovation project that aims at developing a novel reconfigurable modular robotic system able to address some of the challenges related to inspection and maintenance of offshore structures, such as the monitoring of sub-sea foundations of offshore wind parks or the inspection of cage integrity in fish farms. REMORA envisages a modular system consisting of heterogeneous underwater robots that can collaborate and connect physically. By drawing on each other capabilities, the single robots will give rise to a new system with greater functionalities and increased resilience.

At the current stage of development, REMORA includes a set of ROVs of type BlueROV, whose control system has been upgraded to achieve autonomous maneuvering and dynamic positioning in a laboratory environment. The robot dimensions are $[0.483,0.33,0.267] \mathrm{m}$ and its weight is $3.73 \mathrm{~kg}$. Six type T200 ducked propellers provide horizontal 


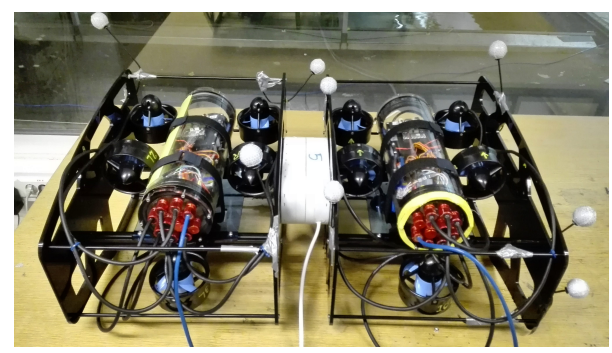

(a) Robots connected through the docking modules.

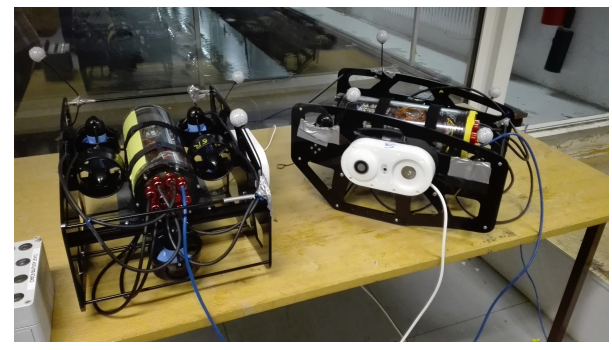

(b) Powering on/off the docking system enables connection and disconnection of the robots.

Fig. 2: Prototype of the REMORA modular system.

and vertical thrust. Each thruster can deliver $50 \mathrm{~N}$ of maximum forward thrust. Electronic speed controllers regulate the power provided to individual thrusters. The basic sensing payload of the robot consists of a 9DOF inertial measurement unit (IMU) and a pressure sensor. The robot is equipped with an Odroid XU-4 for high level data processing and a Pixhawk for low level control. Battery capacity is $6200 \mathrm{mAh}$.

A system for docking is currently under development; this will enable the autonomous connection with tools and the robot-to-robot interconnection. The current prototype of the docking module is physically dimensioned to fit in size the BlueROV and it uses a magnetic-based mechanism to enable and disable the interconnection. The docking mechanism is designed such that energy is utilized only when the robot needs to disconnect from a tool or another robot [22]. Figure 2 shows the actual prototypes of the REMORA connectors. This study assumes that the mechanism of connection/disconnection is perfectly functioning.

\section{HYDROSTATICS OF UNDERWATER ROBOTS}

This section explains the hydrostatics of underwater vehicles, which is essential for calculation of thrust forces needed during operation and change of morphology. In body coordinates, the center of gravity $\boldsymbol{r}_{\boldsymbol{G}, i}^{b}$ and the center of buoyancy $\boldsymbol{r}_{\boldsymbol{B}, i}^{b}$ for each underwater robot are

$$
\begin{aligned}
\boldsymbol{r}_{\boldsymbol{G}, i}^{b} & =\frac{1}{m_{i}} \int_{V} \boldsymbol{r}^{b} \rho_{m, i}\left(\boldsymbol{r}^{b}\right) d V \\
\boldsymbol{r}_{\boldsymbol{B}, i}^{b} & =\frac{1}{\nabla_{i}} \int_{V} \boldsymbol{r}^{b} \rho_{w} d V
\end{aligned}
$$

where $\rho_{m, i}\left(\boldsymbol{r}^{b}\right), m_{i}$ and $\nabla_{i}$ are the distribution of mass at location $\boldsymbol{r}^{b}$, the total mass and the displaced volume of robot $i$, respectively. $\rho_{w}$ is the density of water. The vector $\boldsymbol{r}^{b}$ is the location in the body-fixed frame $\{b\}$ and origin in $\boldsymbol{O}_{\boldsymbol{b}}$. When the mass of a robot does not change over time, $\boldsymbol{r}_{\boldsymbol{G}, i}^{b}$ will be constant and $r_{B, i}^{b}$ will be constant as long as the robot is fully submerged.

For the cluster of $n_{r}$ connected robots, the geometry in the navigation frame (earth-fixed, North-East-Down coordinates) $\{n\}$ has to be considered. Using $\boldsymbol{\eta}_{\boldsymbol{i}}=[x, y, z, \phi, \theta, \psi]_{i}^{T}$ for the pose of robot $i$, and $\boldsymbol{R}_{n b}^{(i)}$ for the rotation from the body to the navigation frame, then the location of gravity and buoyancy centers for the cluster are, in the navigation frame coordinates,

$$
\begin{aligned}
& \boldsymbol{r}_{\boldsymbol{G}, c}^{n}=\frac{1}{\sum_{n_{r}} m_{i}} \sum_{n_{r}}\left(\boldsymbol{p}_{\boldsymbol{O}, i}^{n}+\boldsymbol{R}_{n b}^{(i)} \boldsymbol{r}_{\boldsymbol{G}, i}^{b} m_{i}\right) \\
& \boldsymbol{r}_{\boldsymbol{B}, c}^{n}=\frac{1}{\sum_{n_{r}} \nabla_{i}} \sum_{n_{r}}\left(\boldsymbol{p}_{\boldsymbol{O}, i}^{n}+\boldsymbol{R}_{n b}^{(i)} \boldsymbol{r}_{\boldsymbol{B}, i}^{b} \nabla_{i}\right) .
\end{aligned}
$$

where $\boldsymbol{p}_{\boldsymbol{O}, i}$ is the vector from a chosen origin of the cluster $O_{c}$ to the local origins $O_{i}$ of the members.

Having determined the $\boldsymbol{r}_{\boldsymbol{G}, c}^{n}$ and $\boldsymbol{r}_{\boldsymbol{B}, c}^{n}$ vectors, the restoring forces and moments can be calculated. The buoyancy force, $\boldsymbol{f}_{b}^{n}=-[0,0, B]^{T}$ with $B=\rho_{w} g \nabla$, acts through the center of buoyancy, where $g$ is the acceleration of gravity. The gravity force, $\boldsymbol{f}_{\boldsymbol{g}}^{n}=[0,0, W]^{T}$ with $W=m g$, acts through the center of gravity.

The generalized restoring [force, moment] ${ }^{T}$ vector $\boldsymbol{g}(\boldsymbol{\eta})$ on the cluster, in the body frame, is

$$
g(\eta)=-\left[\begin{array}{c}
f_{g}^{b}+f_{b}^{b} \\
r_{G}^{b} \times f_{g}^{b}+r_{B}^{b} \times f_{b}^{b} .
\end{array}\right]
$$

The restoring moments are essential since they need to be balanced at each independent cluster or robot to maintain passive attitude stability in the horizontal plane. The vertical force balance makes each underwater robot maintain its depth of below the surface.

A neutrally buoyant underwater vehicle satisfies $W=B$. Hence, $\boldsymbol{f}_{g}^{b}=-\boldsymbol{f}_{b}^{b}$.

\section{HYDRODYNAMICS OF UNDERWATER ROBOTS}

This section describes the hydrodynamics, which is essential for motion and reconfiguration control. Viscosity is the main consumer of energy when an underwater robot makes relative speed through the water.

The general equations describing the dynamics of underwater vehicles were formulated in matrix-vector notation by Fossen [23]. Both the disconnected robot and the cluster of connected robots follow these non-linear equations of motion, in the body frame, for kinematics

$$
\dot{\eta}=J(\eta) \nu
$$

and for dynamics

$$
M \dot{\nu}+D(\nu) \nu+g(\eta)=\tau
$$


$\boldsymbol{\eta}=[x, y, z, \phi, \theta, \psi]^{T}$ refers to the pose in the North-EastDown (NED) navigation frame $\{n\}$.

$\boldsymbol{\nu}=[u, v, w, p, q, r]^{T}$ refers to the linear and angular velocity vector in the body frame $\{b\}$.

$M=M_{R B}+M_{A}$ is the generalized inertia matrix, consisting of the rigid-body mass matrix, $M_{R B}$, and the added mass matrix, $\boldsymbol{M}_{\boldsymbol{A}}$.

$D(\nu)=D_{V}(\nu)+D_{N}(\nu)$ is the damping matrix, consisting of the linear damping, $D_{V}(\nu)$, and the quadratic damping, $\boldsymbol{D}_{N}(\nu)$.

$\boldsymbol{J}(\boldsymbol{\eta})$ allows for transformations from $\{b\}$ to $\{n\}$ and it is defined as

$$
J(\eta)=\left[\begin{array}{cc}
T_{b}^{n}\left(\Theta_{n b}\right) & 0_{3 \times 3} \\
0_{3 \times 3} & R_{\Theta}\left(\Theta_{n b}\right)
\end{array}\right]
$$

where $\Theta_{n \boldsymbol{b}}=[\boldsymbol{\phi}, \boldsymbol{\theta}, \boldsymbol{\psi}]^{T}$ refers to the Euler angles, $T_{b}^{n}\left(\Theta_{n b}\right)$ and $R_{\Theta}\left(\Theta_{n b}\right)$ are the linear and angular velocity transformation matrices, respectively.

$\tau=[X, Y, Z, K, M, N]^{T}$ refers to the forces and moments influencing the robot in $\{b\}$.

Note that ocean currents are neglected.

The hydrodynamic matrices described above can be analytically derived by applying strip theory [24], [25], both for the disconnected robot and the cluster of connected robots.

\section{ENERGY CRITERION}

\section{A. Thruster Generated Forces, Moments and Power Con-} sumed

For robots equipped with thrusters, the direction of force from thruster $t h$ is described by the directional cosine vector ${ }^{t h} \boldsymbol{e}$ and by its position vector ${ }^{t h} l$, in the body frame, with respect to the center of geometry $\boldsymbol{O}_{b}$. With thrust command $\boldsymbol{u}$ to the set of thrusters, the forces and moments vector $\boldsymbol{\tau}$ produced is defined as

$$
\boldsymbol{\tau}=\boldsymbol{T} \boldsymbol{u}=\left[\begin{array}{ccc}
{ }^{1} \boldsymbol{e} & \ldots & { }^{n_{t}} \boldsymbol{e} \\
{ }^{1} \boldsymbol{l} \times{ }^{1} \boldsymbol{e} & \ldots & { }^{n_{t}} \boldsymbol{l} \times{ }^{n_{t}} \boldsymbol{e}
\end{array}\right] \boldsymbol{u}
$$

where $n_{t}$ is the number of thrusters and $\boldsymbol{T} \in \mathcal{R}^{6 \times n_{t}}$ is the thruster configuration matrix.

Power consumed by a thruster, $P_{t h}$, is, according to manufacturer's data sheets for a bollard pull, approximately proportional to the square of thrust command. Power consumption of thruster th is, with thrust command $u_{t h}$ and conversion factor $\eta_{t h}$,

$$
P_{t h}=\eta_{t h}\left|u_{t h}\right| u_{t h} .
$$

In practice, thrusters are commanded by a pulse-width modulated signal $u_{P W M}$ and

$$
P_{t h}=P_{t h}^{\max } \frac{u_{P W M}}{u_{P W M}^{\max }} .
$$

When the robot produces speed through water, the obtained thrust decreases, as known from conventional propellers. This effect is not included in the theoretical model, due to lack of open water characteristics for the thrusters.
B. Electric and Mechanical Energy Required to Move from A to $B$

Moving an underwater robot from position $A$ to $B$ along a path $s$ with a velocity profile $\nu$ requires a thrust according to (7). The steady state part of $\tau$ is

$$
\bar{\tau}(s)=D_{V}(\nu(s)) \nu(s)+D_{N}(\nu(s)) \nu(s)+g(\eta(s))
$$

The required mechanical power, $P_{m}$, along the path $s$ is then

$$
P_{m}(s)=\overline{\boldsymbol{\tau}}(s) \frac{d \boldsymbol{s}}{d t}=\boldsymbol{T} \boldsymbol{u} \frac{d \boldsymbol{s}}{d t}
$$

The electric power consumed, $P_{e}$, to produce the required thrust by $n_{t}$ available thrusters is, from (10)

$$
P_{e}(s)=\left(\eta_{t h} \sum_{t h=1}^{n_{t}}\left|u_{t h}(s)\right| u_{t h}(s)\right) \frac{d s}{d t}
$$

The required energy to overcome viscous resistance and compensate for buoyancy force and moments when moving from $A$ to $B$ is then

$$
E_{A B, s}=\int_{A}^{B} P_{e}(s) d s
$$

\section{Thruster Forces and Moments Required for Balancing}

While one robot is swimming, the remaining parts of the cluster need to balance the restoring forces and moments described in (5) by using the remaining thrusters. The thruster forces and moments vector $\tau_{c}$ for the remaining robots is defined as

$$
\boldsymbol{\tau}_{c}=\sum_{j=1}^{n_{r}-1} \boldsymbol{\tau}_{j}
$$

$=\sum_{j=1}^{n_{r}-1}\left[\begin{array}{ccc}{ }^{1} \boldsymbol{e}_{j} & \ldots & { }^{n_{t}} \boldsymbol{e}_{j} \\ \left({ }^{j} \boldsymbol{r}_{0}+{ }^{1} \boldsymbol{l}_{j}\right) \times{ }^{1} \boldsymbol{e}_{j} & \ldots & \left({ }^{j} \boldsymbol{r}_{0}+{ }^{n_{t}} \boldsymbol{l}_{j}\right) \times{ }^{n_{t}} \boldsymbol{e}_{j},\end{array}\right] \boldsymbol{u}_{j}$

where ${ }^{j} \boldsymbol{r}_{0}$ is the center of geometry of robot $j$ and $n_{r}$ is the number of the robots in the system.

Since both force and moment balance need to be obtained simultaneously, the power required to balance is a function of the thrusters geometry available within the cluster and of their positions and orientation.

\section{Energy as Heuristic}

An intuitive heuristic to use for the problem at hand is the energy consumed. The task is to let a robot move from a location $\mathrm{A}$ to a location $\mathrm{B}$, while other robots in one or more clusters use power for compensating the restoring moments, $P_{b a l}$. The electric power, $P_{e}$, is also considered and defined in (14).

The energy required for moving a robot from position $\boldsymbol{A}$ to $\boldsymbol{B}$ along a path $s$, and total duration $T$ is

$$
E_{\text {min }}=\int_{A}^{B} P_{e}(s) d s+\int_{0}^{T} P_{b a l} d t
$$

This is minimized to solve the self reconfiguration problem. 


\section{THE SELF-RECONFIGURATION PROBLEM}

This section provides a formal problem definition.

\section{Given}

g1: A task to perform, requiring specific sensors, actuators or tools to transport.

g2: A cluster of $n_{r}$ underwater robots with different sensing and actuation capabilities to meet the desired task.

g3: A robot $i$ in the cluster with the ability to generate a [force, moment $]^{T}$ vector $\boldsymbol{\tau}^{(i)}=$ $\boldsymbol{T}^{(i)} \boldsymbol{u}^{(i)}$, defined by (9) and constrained by $\boldsymbol{u}_{t h}^{(i)} \subseteq$ $\left\{u_{\min , t h}^{(i)}, u_{\max , t h}^{(i)}\right\}$ for its thruster $t h$.

g4: A robot $i$ in the cluster with the ability to attach magnetically to the other robots and tool(s) by means of its connectors $c^{(i)}$.

g5: A start configuration $\mathcal{S}_{s}$ formed by connections between a robot $i$ and a robot or a tool $j$. The existing connections are described by the adjacency matrix $\boldsymbol{A}_{\boldsymbol{s}}$ defined as

$$
\boldsymbol{a}_{\boldsymbol{i j}}=\left\{\begin{array}{lc}
c^{i} & \text { if } i \text { is connected to } j, \\
0 & \text { otherwise }
\end{array}\right.
$$

g6: A goal configuration $\mathcal{S}_{g}$ formed by connections between a robot $i$ and a robot or a tool $j$. The existing connections are described by the adjacency matrix $\boldsymbol{A}_{\boldsymbol{g}}$ defined in (18).

g7: A suite of services available from a configuration including sensing and actuating capabilities, interaction with tools required during a particular task, and the morphology of the configuration.

g8: A set of conditions $\mathcal{V}$ to satisfy

v1: A robot $i$ at its start location $\boldsymbol{r}_{\boldsymbol{G}, i}^{n}$ in $\mathcal{S}_{s}$ reaches its assigned goal location $\boldsymbol{r}_{\boldsymbol{G}, i}^{n}$ in $\mathcal{S}_{g}$, while the remaining connected robots do not move. The location corresponds to the center of gravity.

v2: A robot $i$ with particular sensing or actuation capabilities is assigned to a goal location specified in the goal configuration description, if needed to complete the task.

v3: While navigating to the goal location, a robot $i$ avoids collisions with the remaining connected robots according to

$$
\boldsymbol{d} \geq \frac{3}{2} \max \left(L_{r}, W_{r}, H_{r}\right)
$$

where $\boldsymbol{d}$ is the distance between the current location and the goal location. $L_{r}, W_{r}$ and $H_{r}$ are, respectively, the length, the width and the height of robot $i$.

v4: It is preserved at least one connection for each tool, if available in the configuration.

v5: Disconnections are not allowed to clear the passage.

v6: The moments about the $x$-axis $(K)$ and about the $y$-axis $(M)$ are balanced to avoid unwanted rotations.

\section{Problem:}

p1: Calculate a sequence of actions including a path from a start location to the assigned goal location, a velocity profile $\nu(t)$ and an order of movements for the robots to self-reconfigure from the start configuration into the goal configuration: $\mathcal{S}_{s} \curvearrowright \mathcal{S}_{g}$ subjected to the set of conditions $\mathcal{V}$.

p2: Minimize the energy criterion defined in (17) and utilize it to guide the Basic Theta* selfreconfiguration algorithm described in Sub-Section VII-A.

\section{A. Basic Theta*}

The environment is represented as a regular $3 \mathrm{D}$ grid with sides along different dimensions defined by $\left|\dot{\boldsymbol{\eta}}_{\boldsymbol{p}}\right|$ Ts. $\dot{\boldsymbol{\eta}}_{\boldsymbol{p}}, p=$ $1,2,3$, is the velocity in the navigation frame, constant in module, and Ts is the time step.

Basic Theta* is a variant of $\mathrm{A}^{*}$. The key difference is that Basic Theta* allows the parent of a location on the grid to be any other location that has line-of-sight to the considered location. In $A^{*}$, the parent must be a visible neighbor. Hence, Basic Theta* finds paths with any potential heading in the continuous environment. $A^{*}$ has heading constrained to grid edges, multiple of 45 degrees. The $\mathrm{A}^{*}$ algorithm is usually coupled with post smoothing techniques, but it is not guaranteed to find truly shortest paths because post smoothing techniques only consider grid edges resulting from the $\mathrm{A}^{*}$ search. On random $500 \times 5002 \mathrm{D}$ grids with $20 \%$ blocked cells, Basic Theta* finds paths shorter than A* with post smoothing techniques $70 \%$ of the time [7]. Nash et al. [8] state that the paths found by $\mathrm{A}^{*}$ on 26-neighbor cubic grids can be $\approx 13 \%$ longer than truly shortest paths. Basic Theta* can be easily extended to 3D grids because it is based on the triangle inequality.

In the present paper, Basic Theta* is adapted to the selfreconfiguration problem to provide a mapping from a given start configuration $\mathcal{S}_{s}$ to a given goal configuration $\mathcal{S}_{g}$, an order of movements for the robots and a path from a start location in $\mathcal{S}_{s}$ to the assigned goal location in $\mathcal{S}_{g}$. The robots move sequentially by following the order of movements specified by the algorithm; therefore, a new intermediate configuration is obtained every time the chosen robot reaches the assigned goal location. The mapping from a start location to a goal location is chosen by minimizing the exact cost function, $g$, and the estimated cost function $h$ (heuristic). Thus, a mapping is formed by robots whose $g+h$ is minimal to one another. The energy criterion described in Section VI is used to guide the self-reconfiguration algorithm. Both $h$ and $g$ include the energy spent to complete the path defined in (15). $g$ also considers the energy needed to balance the restoring moments in (5). Hence, the heuristic is defined such that the estimated path cost is not higher than the exact cost.

\section{RESULTS}

\section{A. Simulations}

Robots to self-reconfigure have at least one connection to a neighbor. The number of connectors mounted on each 


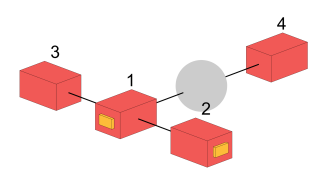

(En:a)

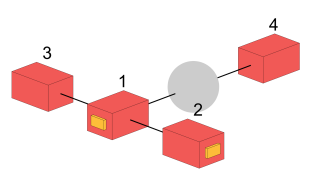

(Eu:a)

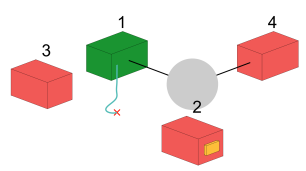

(En:b)

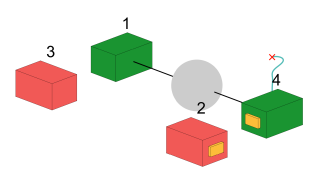

(En:c)

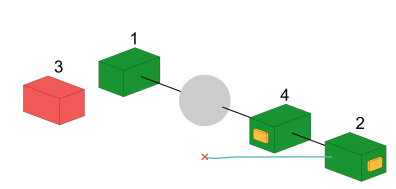

(En:d)

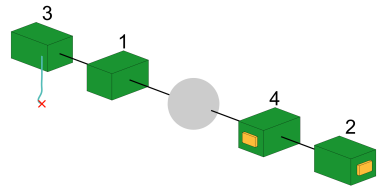

(En:e)

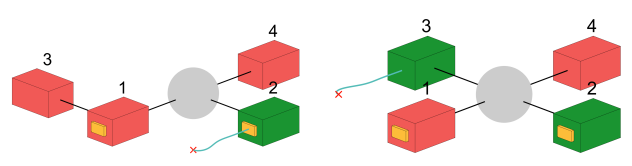

(Eu:b)

(Eu:c)

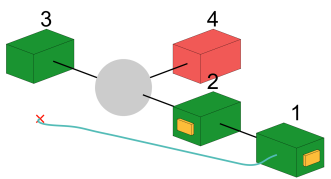

(Eu:d)

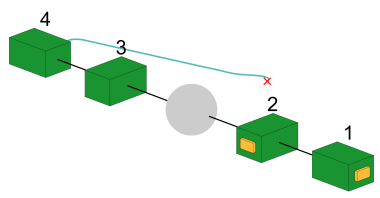

(Eu:e)

Fig. 3: Simulation: Self-reconfiguration by means of the energy criterion (En) and the Euclidean distance (Eu). The robots are represented as boxes coloured in green, if they have already reached the goal location, and in red, otherwise. The yellow box represents a camera. The red cross is the start location already left. The blue line is the path followed. The sphere represents a tool to be used to complete a task.
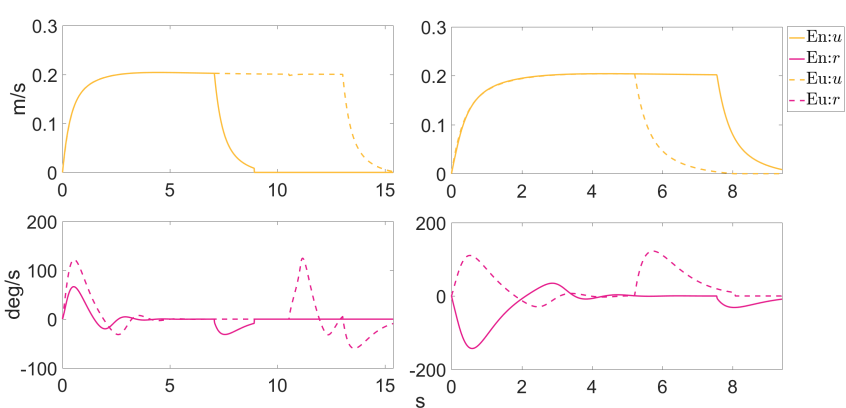

Fig. 4: Simulation: Velocities $u$ and $r$ for navigation (see Section $\mathrm{V}$ for the notation). The first column refers to robot 2 in Figure 3 (En:d) compared to robot 1 in Figure 3 (Eu:d). The second column refers to robot 4 in Figure 3 (En:c) compared to robot 2 in Figure 3 (Eu:b).
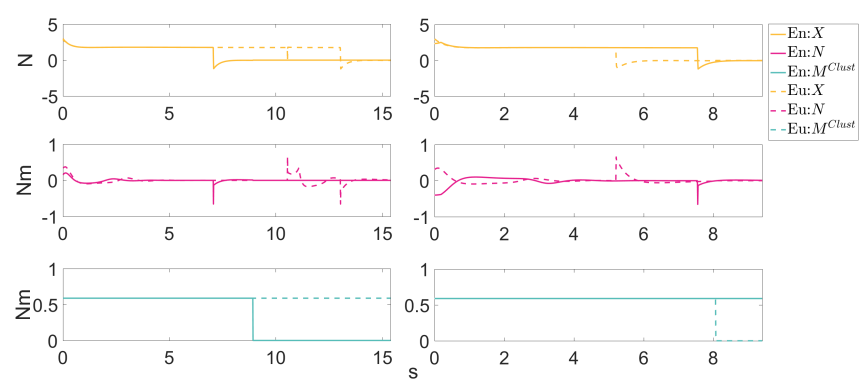

Fig. 5: Simulation: Force $X$ and moment $N$ for navigation, and moment $M$ produced by the hydrostatic moment on the cluster of robots (see Section $\mathrm{V}$ for the notation). The first column refers to robot 2 in Figure 3 (En:d) compared to robot 1 in Figure 3 (Eu:d). The second column refers to robot 4 in Figure 3 (En:c) compared to robot 2 in Figure 3 (Eu:b).

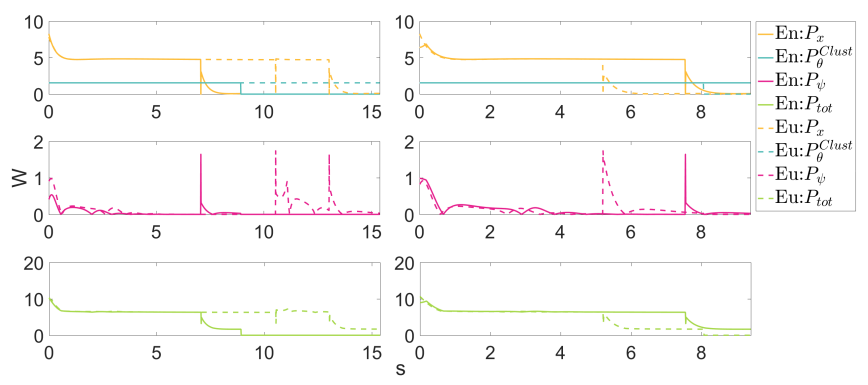

Fig. 6: Simulation: Power consumed to exert the force $X$ and the moment $N$ for navigation, to oppose to the restoring moment $M$ on the cluster of robots and total power. The first column refers to robot 2 in Figure 3 (En:d) compared to robot 1 in Figure 3 (Eu:d). The second column refers to robot 4 in Figure 3 (En:c) compared to robot 2 in Figure 3 (Eu:b).

robot is mapped to the configuration space: the higher the number of connectors, the higher the number of possible configurations. Basic Theta* maps the robots in a start configuration to locations in a goal configuration (see Section VII). The robots move one at time by following a specific order provided by the algorithm while avoiding internal collisions. When its turn comes, a robot disconnects from the configuration, reaches its goal location, then obtains its goal orientation and, finally, connects to the desired robots, if already at the goal location.

Figure 3 (a) shows four robots (red boxes) connected to a tool (sphere) in a T-shaped start configuration. A robot and its camera (yellow box) are neutrally buoyant. The tool is also neutrally buoyant but moves the center of gravity of the connected cluster of robots $3 \mathrm{~cm}$ along the $x$-axis. 


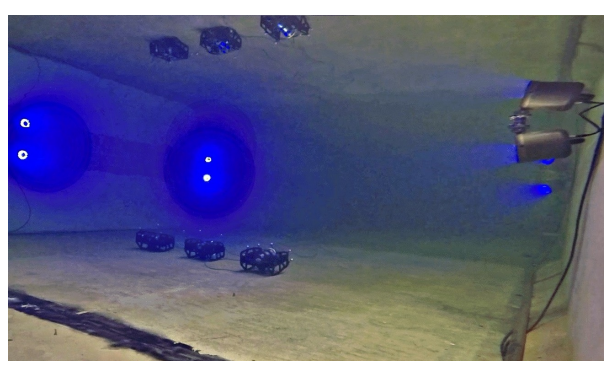

(a)

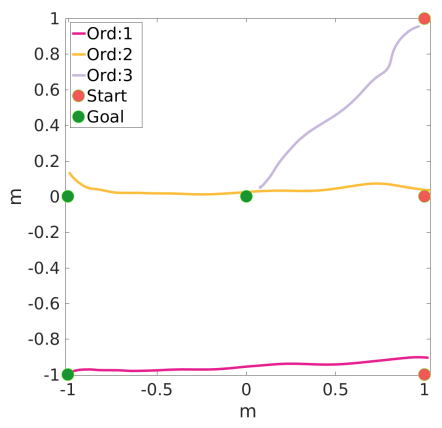

(b)

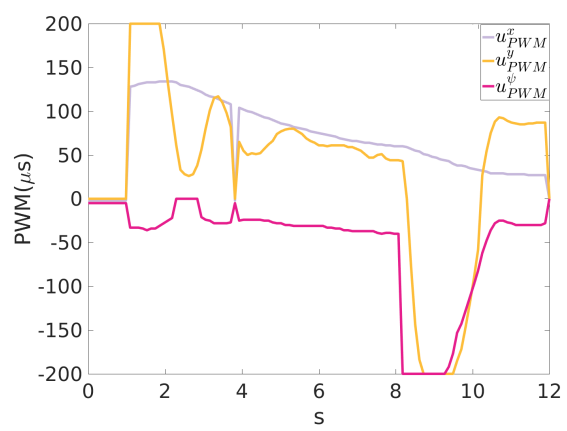

(c)

Fig. 7: Experiment. (a) Three robots deployed as an I-shaped configuration in a [40,6.45,1.5]m model basin. The Qualisys tracking system uses intense light to illuminate the markers on the robots and locate them. (b) From an I-shaped start configuration to an L-shaped goal configuration. The order of movement is specified in the legend. (c) Commanded PWM signals to T200 thrusters for the second robot to move in (b).

This produces a restoring moment $M$ (about the $y$-axis) on the cluster. Requiring to fit a narrow passage, the robots self-reconfigure into the goal configuration in Figure 3 (e). Robots that have already reached the assigned goal location are colored in green. In Figure 3 (En), the robots selfreconfigure according to Basic Theta* guided by the energy criterion described in (17). In Figure $3(\mathrm{Eu})$, the robots self-reconfigure according to Basic Theta* guided by the Euclidean distance. Paths are simulated through a speed controller with the Line-of-Sight (LOS) guidance law for navigation [23]. Curved paths in Figures 3 (Eu:d) and Figure 3 (Eu:e) are due to the bounding box set around each robot to avoid collisions. Table 1 provides the energy and the time spent to have complete self-reconfiguration, both for the energy criterion and for the Euclidean distance. Body velocities (see Figure 4), forces and moments (see Figure 5 ), and power consumed (see Figure 6) for robots assigned to the same goal location by the two different heuristics are also considered. In particular, these figures show robot 2 in Figure 3 (En:d) compared to robot 1 in Figure 3 (Eu:d) and robot 4 in Figure 3 (En:c) compared to robot 2 in Figure 3 (Eu:b). Solid lines (dashed lines) in the figures refer to the results from the energy criterion (Euclidean distance). Figure 4 shows that the energy criterion assigned paths with less change in orientation, so that the moving robots are never in proximity of another robot. Note that the initial and final changes in orientation depend on the desired angles chosen at generation time. Figure 5 shows a constant restoring moment $M$ acting on the cluster of connected robots and introduced by the $3 \mathrm{~cm}$ displacement in the center of gravity of the tool.

TABLE I: Energy (E) and time to self-reconfigure for the energy criterion and the Euclidean distance

\begin{tabular}{ccccc} 
& E to navigate & E to balance & Total E & Time \\
\hline Energy & $150.93 \mathrm{~J}$ & $56.92 \mathrm{~J}$ & $207.86 \mathrm{~J}$ & $36.64 \mathrm{~s}$ \\
Euclidean & $186.96 \mathrm{~J}$ & $68.43 \mathrm{~J}$ & $255.39 \mathrm{~J}$ & $44.0 \mathrm{~s}$ \\
\hline
\end{tabular}

Hence, a moment of $0.6 \mathrm{Nm}$ needs to be balanced for both the solutions but the total power consumed for balancing is lower for the energy criterion. This is because the time needed to self-reconfigure is shorter for the energy criterion (see Table 1). Fast changes in power consumed to produce forces for the moving robot in Figure 6 are due to the robot turning in place, once arrived at the goal location, to achieve the desired orientation.

\section{B. Experiments}

Experiments were conducted in MClab at NTNU, in a $[40,6.5,1.4] \mathrm{m}$ model basin (see Figure 7a). A Qualisys system tracked three BlueROVs that self-reconfigure from an Ishaped start configuration to an L-shaped goal configuration in real time. Five markers were taped asymmetrically on each robot to enable the Qualisys system to track all robots and to calculate their pose. A path controller used a Line-of-Sight (LOS) guidance law [23] for heading control and provided thrust to navigate the desired robot.

Information was transferred to the robots via cables in the water. Some cores in each cable comprised connectdisconnect commands to the docking mechanisms. However, the use of the tethered connectors resulted in robots entangled with cables. Thus, they were not included in this experiment.

The self-reconfiguration algorithm described in Section VII mapped the start locations to the goal locations, found waypoints to navigate to the goal locations and an order of movements for the robots (see Figure 7b). Robots were able to navigate to the goal location with reasonable accuracy in the basin. However, the basin positioning accuracy does not suffice for autonomous docking, which require quite narrow tolerances. The final version of the docking module will solve this issue.

The power consumed for one robot navigating to the assigned goal location is shown in Figure 7c. It is obtained by mapping the recorded pulse-width modulated signals (PWM), sent to the thrusters, to the corresponding electric power as stated in (11). A static mapping, a bollard pull at 
zero speed through water, is available in the BlueRobotics specifications for T200 thrusters. Recent model tests provide true propeller characteristics that could be used to get a much better estimate of power consumption when the robots make speed through water [26].

In real environments, autonomous localization would need to be integrated in each robot. Solutions based on onboard camera image processing and imaging sonar exist [27],[28],[29], where localization can be made possible by partial knowledge of geometry of man made structures or landmarks.

\section{CONCLUSION}

This paper investigated self-reconfiguration of heterogeneous modular underwater robots that have capability of autonomous physical connection. The Basic Theta* algorithm was extended with an energy heuristics and was used to calculate the order of vehicle movements to change from a start to an end morphology. Energy to move vehicles and to balance restoring moments were considered. Basic Theta* favored straight paths to curved paths and chose intermediate configurations with low restoring forces. A comparison with Basic Theta* guided by the Euclidean distance showed the energy heuristic to be superior in this underwater application. A preliminary experiment with model basin selfreconfiguration was also presented.

Future directions of research of the self-reconfiguration problem offer several interesting topics such as using variable vehicle speed in the optimization; allowing robots to move in parallel to reduce time and energy; including docking maneuvering energy in the cost function; generating optimal configurations to meet task specific requirements. Hardware improvements could include wireless communication, e.g. LED based signaling.

\section{ACKNOWLEDGEMENTS}

We gratefully acknowledge support from the REMORA development team at DTU and from the NTNU Centre for Autonomous Marine Operations and Systems (AMOS), to which the Research Council of Norway is the main sponsor (grant 223254).

\section{REFERENCES}

[1] I. Vasilescu, C. Detweiler, M. Doniec, D. Gurdan, S. Sosnowski, J. Stumpf, and D. Rus, "AMOUR V: A hovering energy efficient underwater robot capable of dynamic payloads," The International Journal of Robotics Research, vol. 29, no. 5, pp. 547-570, 2010.

[2] S. Mintchev, E. Donati, S. Marrazza, and C. Stefanini, "Mechatronics design of a miniature underwater robot for swarm operations," in IEEE Proc. Int. Conf. Robotics and Automation (ICRA), 2014.

[3] T. Schmickl, R. Thenius, J. Timmis, A. Tyrrell, J. Halloy, C. Stefanini, L. Manfredi, A. Campo, D. Sutantyo, and S. Kernbach, "CoCoRo: The self-aware swarm of underwater robots," IEEE/RSJ Proc. Int. Conf. Intelligent Robots and Systems (IROS), 2011.

[4] M. Yim, W.-M. Shen, B. Salemi, D. Rus, M. Moll, H. Lipson, E. Klavins, and G. Chirikjian, "Modular self-reconfigurable robot systems [Grand challenges of robotics]," IEEE Robotics \& Automation Magazine, vol. 14, no. 1, pp. 43-52, 2007.

[5] K. Stöy, D. Brandt, and D. J. Christensen, Self-reconfigurable robots: an introduction, ser. Intelligent robotics and autonomous agents. Cambridge, Mass.: MIT Press, 2010.
[6] S. Murata and H. Kurokawa, "Self-reconfigurable robot: Shapechanging cellular robots can exceed conventional robot flexibility," IEEE Robotics \& Automation Magazine, 2007.

[7] A. Nash, K. Daniel, S. Koenig, and A. Felner, "Theta*: Any-angle path planning on grids." Journal of Artificial Intelligence Research, vol. 39, pp. 533-579, 2010.

[8] A. Nash and S. Koenig, "Any-angle path planning," Artificial Intelligence Magazine, vol. 34, no. 4, pp. 85-107, 2013.

[9] Y. Mei, Y. Lu, Y. Hu, and C. Lee, "Energy-efficient motion planning for mobile robots," in IEEE Proc. Int. Conf. Robotics and Automation (ICRA), 2004.

[10] T. Wang, B. Wang, H. Wei, Y. Cao, M. Wang, and Z. Shao, "Stayingalive and energy-efficient path planning for mobile robots," in Proceedings of the American Control Conference (ACC), 2008.

[11] S. Liu and D. Sun, "Minimizing energy consumption of wheeled mobile robots via optimal motion planning," IEEE/ASME Transactions on Mechatronics, vol. 19, no. 2, pp. 401-411, April 2014.

[12] A. Pal, R. Tiwari, and A. Shukla, Soft Computing Techniques in Vision Science. Springer, 2012, ch. Modified A* Algorithm for Mobile Robot Path Planning, pp. 183-193.

[13] D. Brandt, "Comparison of $\mathrm{A}^{*}$ and RRT-connect motion planning techniques for self-reconfiguration planning," in IEEE/RSJ Proc. Int. Conf. Intelligent Robots and Systems (IROS), 2006.

[14] L. De Filippis, G. Guglieri, and F. Quagliotti, "Path planning strategies for uavs in 3d environments," Journal of Intelligent \& Robotic Systems, vol. 65 , pp. $247-264,2012$.

[15] C. Detweiler, S. Sosnowski, I. Vasilescu, and D. Rus, Experimental Robotics. Springer, Berlin, 2009, vol. 54, ch. Saving Energy with Buoyancy and Balance Control for Underwater Robots with Dynamic Payloads, pp. 429-438.

[16] I. O’Hara, J. Paulos, J. Davey, N. Eckenstein, N. Doshi, T. Tosun, J. Greco, J. Seo, M. Turpin, V. Kumar, and M. Yim, "Self-assembly of a swarm of autonomous boats into floating structures," in IEEE Proc. Int. Conf. Robotics and Automation (ICRA), June 2014.

[17] J. Paulos, N. Eckenstein, T. Tosun, J. Seo, J. Davey, J. Greco, V. Kumar, and M. Yim, "Automated self-assembly of large maritime structures by a team of robotic boats," Automation Science and Engineering, IEEE Transactions on, vol. 12, no. 3, pp. 958-968, July 2015.

[18] J. Seo, M. Yim, and V. Kumar, "Assembly planning for planar structures of a brick wall pattern with rectangular modular robots," in IEEE Proc. Int. Conf. Automation Science and Engineering (CASE), Aug 2013, pp. 1016-1021.

[19] S. Mintchev, C. Stefanini, A. Girin, S. Marrazza, S. Orofino, V. Lebastard, L. Manfredi, P. Dario, and F. Boyer, "An underwater reconfigurable robot with bioinspired electric sense," in IEEE Proc. Int. Conf. Robotics and Automation (ICRA), May 2012, pp. 1149-1154.

[20] M. J. Doyle, X. Xinyu, G. Yue, F. Perez-Diaz, C. Parrott, and R. Groß, "Modular hydraulic propulsion: A robot that moves by routing fluid through itself," in IEEE Proc. Int. Conf. Robotics and Automation (ICRA), 2016, pp. 5189-5196.

[21] R. Fitch, "Heterogeneous self-reconfiguring robotics," Ph.D. dissertation, Dartmouth College, 2004.

[22] D. Christensen, J. Andersen, M. Blanke, L. Furno, R. Galeazzi, P. N. Hansen, and M. Nielsen, "Collective modular underwater robotic system for long-term autonomous operation," ICRA Workshop on Persistent Autonomy for Aquatic Robotics: the Role of Control and Learning in Single and Multi-Robot Systems, 2015.

[23] T. Fossen, Handbook of Marine Craft Hydrodynamics and Motion Control. Wiley, 2011.

[24] J. Newman, Marine Hydrodynamics. Cambridge, Mass.: MIT Press, 1977.

[25] O. Faltinsen, Sea Loads on Ships and Offshore Structures. Cambridge University Press, 1990.

[26] M. C. Nielsen, O. A. Eidevik, M. Blanke, and I. Schjølberg, "Constrained multi-body dynamics for modular underwater robots - theory and experiments," 2017, to be published.

[27] J. Jung, J.-H. Li, H.-T. Choi, and H. Myung, "Localization of AUVs using visual information of underwater structures and artificial landmarks," Intelligent Service Robotics, vol. 10, no. 1, pp. 67-76, 2017.

[28] D. Ribas, P. Ridao, J. Neira, and J. Tardos, "Slam using an imaging sonar for partially structured underwater environments," in IEEE/RSJ Proc. Int. Conf. Intelligent Robots and Systems (IROS), 2006.

[29] F. Bellavia, M. Fanfani, and C. Colombo, "Selective visual odometry for accurate auv localization," Autonomous Robots, vol. 41, no. 1, pp. 133-143, 2017. 